\title{
A Digital Bulletin Board for the School Community
}

\author{
Panagiotis Karageorgos, and Dimitrios Gouvedaris
}

\begin{abstract}
This text describes the necessity of having a digital bulletin board in the school environment. In the context of the implementation of the project, this document also describes in detail the hardware and software specifications, the proposed use and the expandability of a wireless and remote programmable digital board, also referred as DPVW (Distance Programmable Video Wall). This paper aims to provide a proposal for a universal use of digital boards for informational use of a school community, students, teachers and school staff.
\end{abstract}

Index Terms-Bulletin Board, School, Digital, Distance Programmable Video Wall, Raspberry Pi.

\section{INTRODUCTION}

In the school environment, many announcements are posted or need to be posted in time. School bulletin boards today, usually consist of a piece of cork with a wooden frame and dimensions of $45 \times 60 \mathrm{~cm}$ or larger. They feature thick-headed pins in transparent colors, ropes and brackets. They are placed at a distinct point in the school building. Announcements are in paper form and are pinned to them addressing to the school community, teachers and/or students.

From our experience, of over 20 years in school community, we can say that this method has proven not effective enough over time, with the result that community information is not working properly.

Many of the announcements are not read because they are posted at irregular time intervals or may be difficult to read, because they are dense, or written in small fonts. Some others are posted in a multi-page form, and only the first page can be clearly viewed or may be other paper messages are posted over them etc. From our experience also, we can say that, some members of the school community do not pay much attention to announcements in such traditional bulletin boards.

Trying to give a solution to this problem we developed a digital bulletin board consisting of colored led panel pieces. These panels may be connected together in a serial form and give to our digital bulletin board the dimensions we prefer as shown in Fig.1 below.

So, we can give to the matrix the required dimensions, to cover the desired area that we want, and make it easier to display text messages or even image or video in it.

The only limitation in the serial connection of the panels relates to the required electric current and of course to the power supply. For example, to connect serial four (4), with the dimensions of $64 \times 32$ led-panels, is required a power supply that gives $10 \mathrm{Amps}$ in $5 \mathrm{~V}$ DC of electric current.

Published on February 17, 2020.

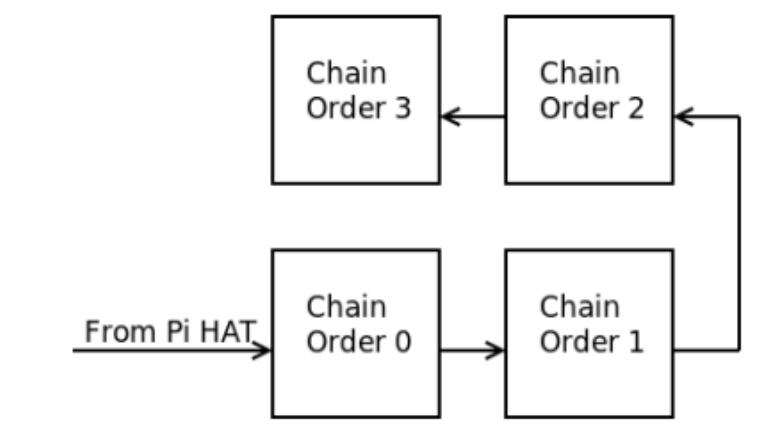

Fig. 1. Example of a 4 panel led square display might be connected as above

\section{IMPLEMENTING THE PROJECT}

\section{A. Development phases}

The implementation of the project took into account all the necessary parameters that would ensure sustainability in the context of the operation of a school unit.

Thus, the phases were performed in a time order as described below.

1) Feasibility study - Analysis and recording of needs.

Initially, took place the process of identifying problems and opportunities, targeting, finding appropriate solutions, and weighing the disadvantages and benefits of the various alternatives for the implementation. In addition, market analysis and research was carried out as well as the recording of the equipment necessary for the creation of the Digital Programmable Video Wall (DPVW). Also, the technical viability analysis and the cost-benefit analysis were designed to produce documented conclusions as to whether it is technically and economically advantageous, to implement the specific project for the creation of a digital bulletin board in the particular school unit.

2) Hardware and material supply - Initial Installation.

Initially, the implementation of the project required: a Raspberry Pi 3 Model B, 5V Raspberry Pi 3 Power Supply, one MicroSD 16GB, a Matrix Led Display 64x32, Power Supply 5Amp for the LED MATRIX DISPLAY (LMD), Cable Adapters, an Adafruit RGB Matrix HAT. We have also been supplying any additional consumable materials that have been specified as necessary. Then all necessary connections between hardware parts were implemented and the system was tested for reliability and also response and failure tests have been made.

3) Writing code using the Python programming language We used python code to test the display of messages on the small Matrix Led Display 64x32.

4) In this phase, the DPVW consisting of the Raspberry Pi, the HAT and the LMD was wirelessly connected to an access point within the school unit, and tested, in order 
to realize the communication that allows remote programming of the matrix.

5) An application has been implemented on devices with Android and/or IOS operating systems on mobile phones and /or tablets for the purpose of programming automatic display of messages in the LMD such as announcements for the school community by the head of the school unit or the school stuff responsible for communication. Therefore, code writing with the Python programming language is required.

Also, the interface was implemented in a PHP language environment with javascript.

6) Upon successful completion of previous "phase 5", additional hardware was provided and further installation needed was accomplished in order to extend the implementation. The further hardware and the materials needed was about the:

- Extension of LMD. This was done by purchasing one or more led matrices that could be serially connected to increase the viewing area of the LMD. We managed to do this extension by connecting in a serial way two (2) $64 \times 32$ led matrices.

So we had constructed a $128 \times 32$ LMD. Theoretically much more matrices may be connected like this.

Each matrix has 2048 bright RGB LEDs arranged in a $64 \times 32$ grid on the front. On the back there is a PCB with two IDC connectors (one input, one output: in theory you can chain these together) and two 16-bit latches that allow you to drive the display with a 1:16 scan rate.

These displays are technically 'chainable' - connect one output to the next input - It requires a high speed processor and more RAM than the Arduino has! That is why a Raspberry $\mathrm{Pi} 3$ is the most appropriate hardware to drive one or many matrices. [1]

- Creation of a timber case, able to fit and support the entire structure. This, must be a stable and durable construction. We also took note to have some protection against natural disasters that could destroy or break the led matrices, by fitting a transparent Plexiglas in the front of the case.

- Extension of the electrical installation to an appropriate location within the school unit building, by a qualified electrician, to fit the LMD with the all other components (raspberry pi, power supply, construction brackets etc.). Then, the installation and operation test was performed.

7) As part of the dissemination of the project's results, a briefing was held, a presentation was made and a blog with full description of the phases and photographic material was created at the internet.

\section{B. User Interface Architecture}

The following were taken into account when developing the interface environment:

1) The use and management of the Digital Bulletin Board (or DPVW) will be done by users without specific technological knowledge.

2) The use-menu must be accessible from all cordless mobile devices (laptops, cell phones, tablets) within the school unit area.
3) The use-menu should only be used and managed by authorized users, thus creating a security system that requires a password was necessary. It should be noted here also, that the DPVW is placed to a position into the school building so that, it is clearly visible and so that, messages displayed on it, can be easily read by school unit staff, students and teachers.

As already mentioned the development of the interface and management environment was done using the Hypertext Preprocessor (PHP) programming language, javascript and python script in the part of sending messages from the interface to the LMD.

The hardware driving of the LMD was implemented by using a HAT in conjunction with Raspberry Pi 3+. [2, [3]

The implementation of the interface provides the user at first, a menu with two (2) basic options. The DPVW user can choose between two types of messages to be displayed on the LED MATRIX DISPLAY(LMD), the "scrolling messages" and/or the "rotating pages".

If a message is scrolling, then it is displayed from the right side to the left of the matrix until the whole is displayed. The message is entered in 1 to 4 lines and each line can be scrolling or steady.

"Rotating pages" messages are initially displayed throughout the matrix area for a short period of time until they are read by those interested in a page format and then erased. The message will continue to display to the next page again for a short period time to read, and so on, and the flow will continue until the entire message is viewed. See the figure below:

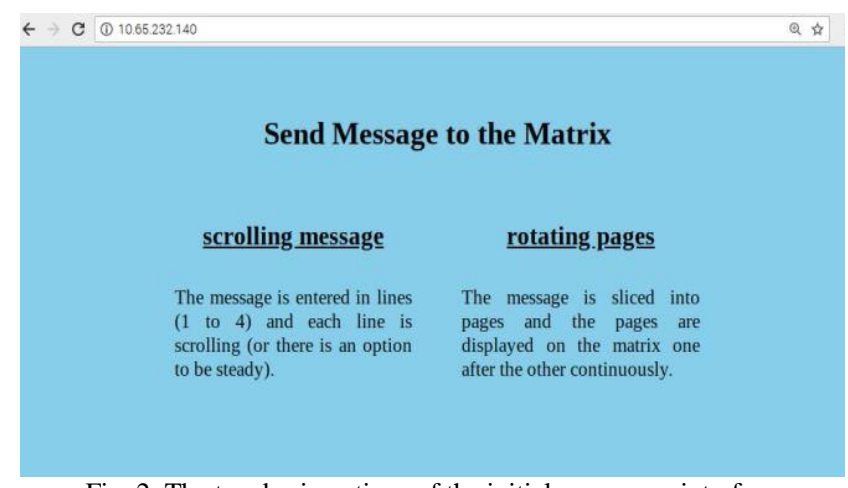

Fig. 2. The two basic options of the initial user menu interface

Depending on the type of message, the user can have different sub-menu screens with the following sub-options:

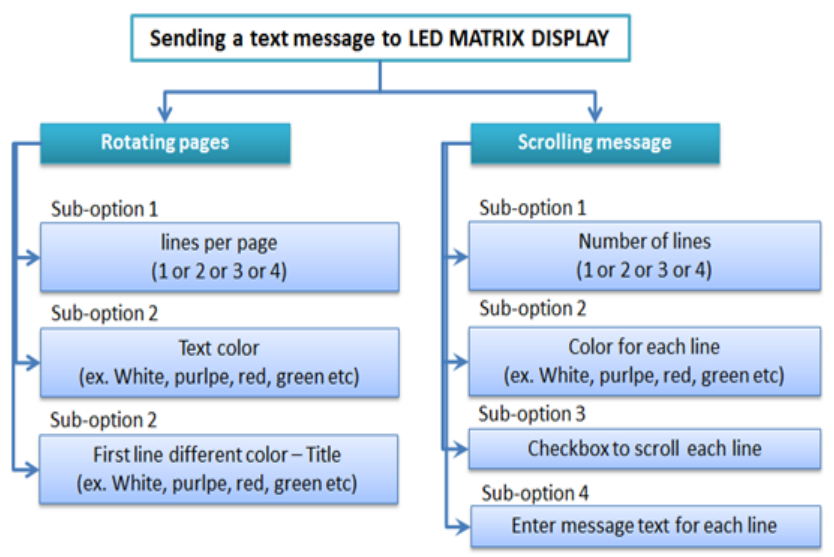

Fig. 3. The user can choose between several sub-options depending on the type of message to display 
By choosing the "Scrolling message" option, the user can have a sub-menu screen with the following sub-options:

- The sub-option "number of lines" is representing the number of lines for the displayed message. This can be between 1 to 4 . The number of lines is limited to 4 , because for a $128 \times 32 \mathrm{LMD}$, as we constructed, the message must be big enough to be viewable from a distance of about 10 meters from any observer.

- With the sub-option "color for each line", theoretically the user can choose between any color from the RGB pallet. But for the convenience of the user only the 16 basic colors can be chosen by their names from a dropdown menu.

- There is also a checkbox "scroll" that can allow if checked, each line to be scrollable or steady.

- Finally, a textbox "message" for each line can be filled with text that will represent the displayable text message for that line.

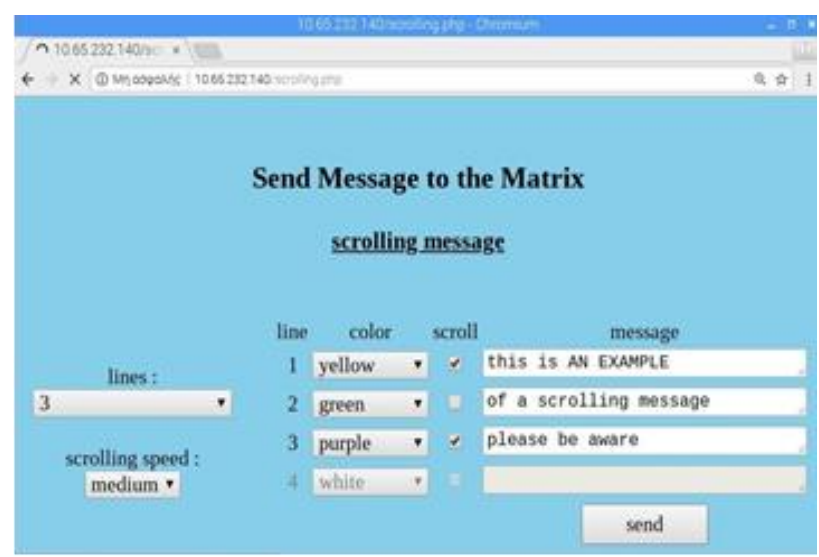

Fig. 4. The "scrolling message" sub-menu

By choosing the "rotating pages" option, the user can have a sub-menu screen with the following sub-options:

- The sub-option "lines per page" can be a number not greater than 4 since the message in the $128 \times 32$ LMD as we constructed must be big enough, to be viewable from a distance of about 10 meters from any observer.

- With the sub-option "Text color" the user can choose between the 16 basic colors of the RGB pallet by their names from a dropdown menu.

- With the sub-option "First line different color" a different color for the first line of message can be chosen. This sub-option will emphasize the first line of the text displayable message, if the user wants to display something like a title to his message.

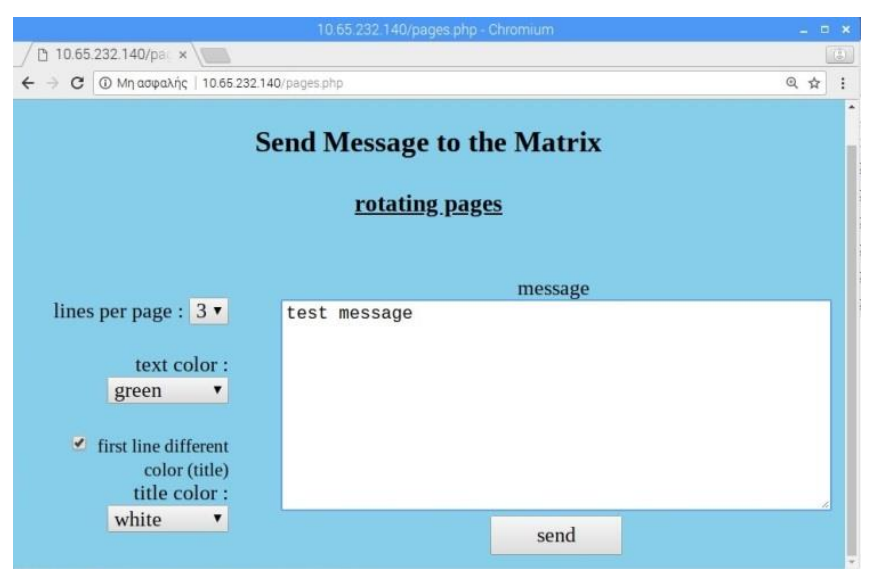

Fig. 5. The "rotating pages" sub-menu

\section{Coding}

Most of the examples provided with the driving software are using libraries preinstalled [4], but familiarity with programming languages is essential for project implementation.

Although installing the required software modules in the Raspberry environment provides several examples written in languages such as $\mathrm{C}++$ and Python that allow key messages to be sent to the LMD, creating an environment where a simple user can send his own messages is a job for programmers.

The user interface can be programmatically implemented in two ways primarily. As an interface in the form of a Web site that requires additional installation of a Web Server and SQL Server in the Raspberry Pi.

Web server pages with user menu options will be accessible wirelessly from the local school network and should be in a dynamic form (eg. written in PHP and Javascript format), thus to interact live with the software driving matrices commands, and for the user to see when sending a message, at the same time, this to appear into the LMD.

Another way to implement this is by using a programming environment like Android Studio [5] that can easily support and create the connection between the Raspberry Pi and Android OS mobile devices and make the interaction happen.

The following example code is an image-scroller class written in python programming language.

This can be executed from command line with an argument (-i or - - image) for an image. The image is scrolling to the LMD each time by moving one step (expressing with the xpos variable). The image format is PPM. The PPM format is a lowest common denominator color image file format. Anyone can create this kind of images using a free software for image processing such as Gimp [6].

Another argument when calling from command line in this example is "chain" (- -chain=). With this argument the caller can pass a clear number representing the number of matrices chained in the LMD. 


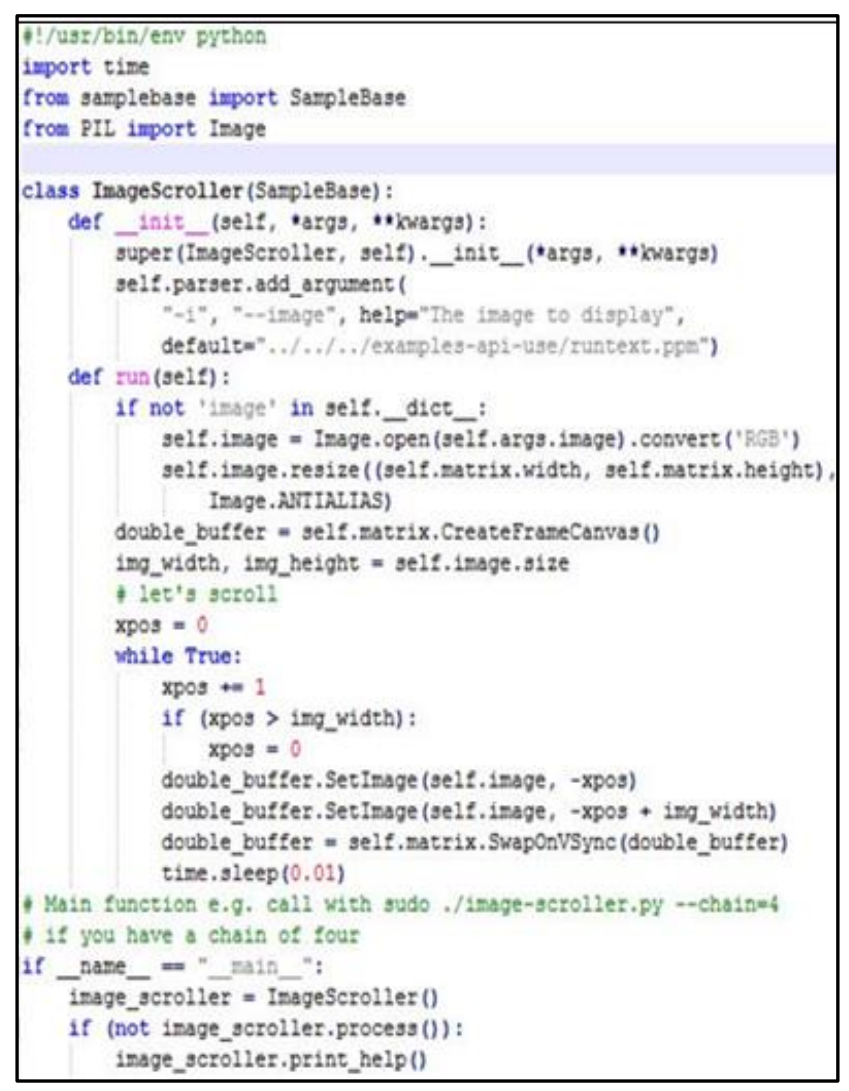

Fig. 6. The ImageScroller class in python

\section{THE DPVW AS AN EDUCATIONAL TOOL IN SCHOOL - BENEFITS}

Computational Thinking is a problem solving approach geared toward understanding and learning in the $21^{\text {st }}$ Century [7].

The DPVW that we are describing here, is a high level Computational Thinking problem in technology-supported learning, and can easily be used to school, as an experiential approach for teaching programming and connectivity between systems using different operating systems such as linux $($ Raspbian $=$ Raspberry + Debian $)$ for Raspberry Pi and Android for mobile devices, tablets etc.

Also the Raspberry Pi computer, that this project is based to, is a perfect fit for the maker movement and its associated learning theories [8]. It is accessible to young learners, while still providing enough challenge to embrace learners at all levels. It encourages the construction of both mental and physical models, namely through computer programming/coding and the construction of electronic circuits.

The Raspberry Pi in technology-supported learning for middle class students, is motivating the development of new curricula in control education and the internet of things [9].

Another benefit is that python programming language, which is mostly used in our project, is a text-based, high level and classic, computer programming language designed to be relatively easy for beginners [10].

\section{EXTENDING THE PROJECT - PERSPECTIVES}

An analysis of the methodology of applying the project to more school units and how it is expected to achieve possible collaboration with administrative units, ministries and bodies or even professionals may be feasible.

The affordable cost of implementing the project could allow for the expansion and creation of this construction in many places.

Thus, for example, the implementation of a centrally managed DPVW network could provide the prospect of immediate and timely information for school staff in many units.

Messages that could deal with organizational issues and/or early warning issues (ex. evacuation of buildings due to imminent natural disasters) could be sent from a central administration of the network.

At the same time having a closed VPN network from DPVW or digital messaging boards, within an organization or ministry is the best insurance for privacy, security and validity of messages [11].

On the other hand, if one considers the possibilities of extending the LMD, then he could imagine creating a large enough Video Wall to allow the viewing of movies or complete programs. Essentially, creating a large DPVW requires only one Raspberry Pi with $\mathrm{HAT}$, and the main limitation in the linkage chain between the matrices, is the power supply.

\section{ONLINE RESOURCES AND PHOTOS}

The following photographs depict the front and back of the DPVW construction. Related videos describing the project's implementation have been posted on YouTube [12]. For this reason, also a related blog has been created [13].

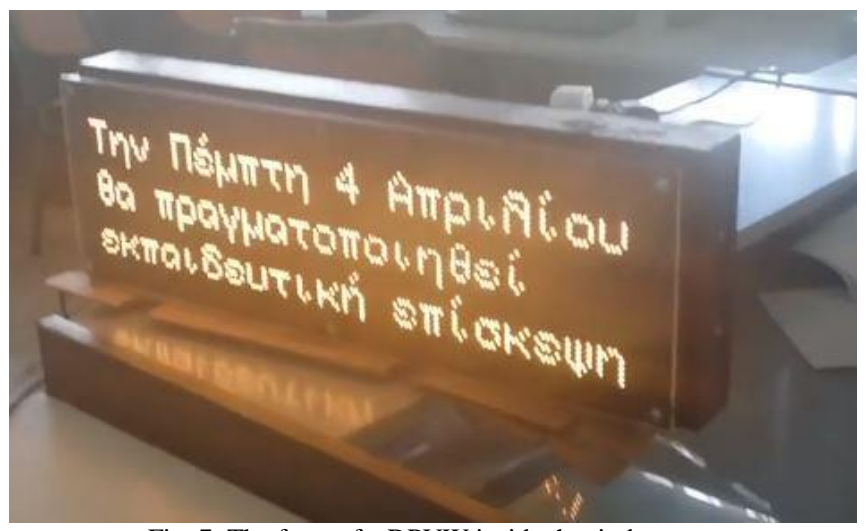

Fig. 7. The front of a DPVW inside the timber case

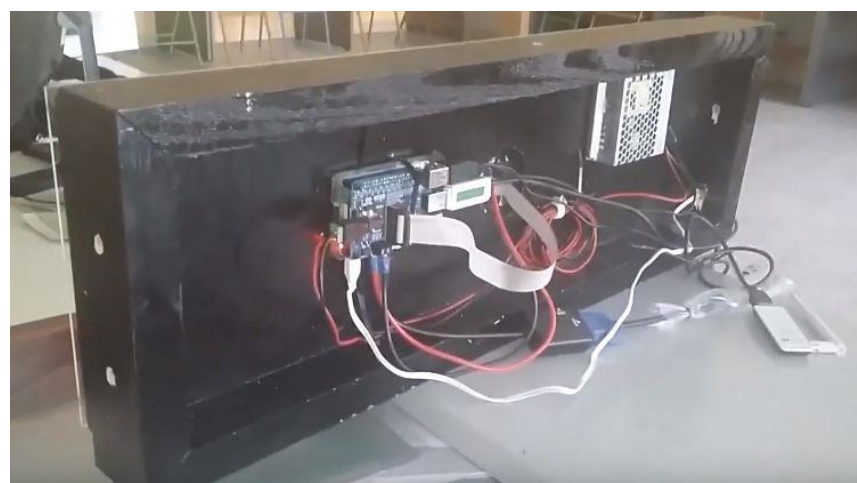

Fig. 8. The back side of a DPVW 


\section{REFERENCES}

[1] Description of the $4 \mathrm{~mm}$ pitch $64 \times 32$ RGB LED Matrix. Available at https://www.adafruit.com/product/2278

[2] Adafruit RGB Matrix + Real Time Clock HAT for Raspberry Pi. Available at https://learn.adafruit.com/adafruit-rgb-matrix-plus-realtime-clock-hat-for-raspberry-pi/driving-matrices.

[3] Raspberry Pi 3 is out now! Specs, benchmarks \& more - The MagPi Magazine the MagPi Magazine. Available at https://www.raspberrypi.org/magpi/raspberry-pi-3-specsbenchmarks/

[4] Documentation accompanying hzeller's code repository in github. Available at https://github.com/hzeller/rpi-matrix-pixelpusher

[5] Android Studio. Available https://developer.android.com/studio/intro/index.html

[6] A PPM image. Available http://netpbm.sourceforge.net/doc/ppm.html

[7] Computational thinking by GOOGLE. Available at http://computationalthinkingcourse. withgoogle.com/unit?lesson=8\&u nit $=1$

[8] Constructionism, a Learning Theory and a Model for Maker Education - Stanford University. Available at: http://fablearn.stanford.edu/fellows/blog/constructionism-learningtheory-and-model-maker-education

[9] V. Callaghan, "Buzz-boarding; practical support for teaching computing, based on the internet-of-things," 1st Annual Conference on the Aiming for Excellence in STEM Learning and Teaching, Imperial College, London \& The Royal Geographical Society, 2012.

[10] Alex Bradbury, Ben Everard "Learning Python with Raspberry Pi" ISBN13: 978-1118717059 "Wiley", 2015, pp 23

[11] Tripti Sharma, Rahul Yadav "Security in Virtual private network". Available at: https://pdfs.semanticscholar.org/6447/395a2f7649ec609aabdd28863c 506d3d9399.pdf, International Journal of Innovations \& Advancement in Computer Science, 2015

[12] Video posted in YouTube describing the project implementation and the result. (1) https://youtu.be/Acu-qsqrfn0. https://youtu.be/UVODgvL1fvQ

[13] Blog with publications from the implementation of the project in a Greek Technical School, Available: https://noticeboardschool.blogspot.com/

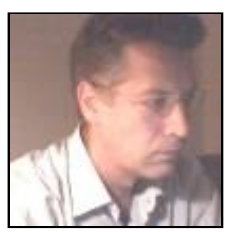

Professional.

\section{Now, work} training of High School I.T. professors/trainees for the Greek National Ministry of Education \& Diofantos. He is General Secretary of the Organizing Committee for the National Informatics Competition for students in Greece, also vice-president in the non-profit organization Greek Computer Society (EPY).

At the past he worked as Programmer-Analyst for 6 years in National Management and Organization (NMO), and 3 years to other small software companies. He has publications in Greek Conference on Informatics in Education, on Informatics / Workshop on Informatics in Education, and as writer in books for computer science and school aids.

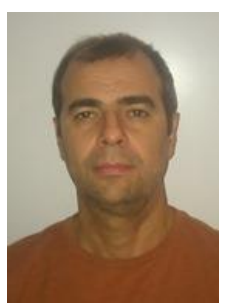

Dimitrios Gouvedaris was born in Edessa, Greece in 1974. He obtained his diploma in Computer Engineering and Informatics from University of Patras in 1998.

He had been a member of the High Performance Computing Laboratory of the Computer Engineering and Informatics Department in Patras for three years (1998-2001). He has been working as Secondary Education Informatics teacher for the Greek Ministry of National Education since 2002. He is proficient in programming languages in local $\mathrm{PC}$ environments such as $\mathrm{C}++$, Python. 